\title{
Neutron detector development for microsatellites
}

Julia G. Bodnarik, Dave K. Hamara, Arnold Burger, Vladimir Buliga, Joanna C. Egner, et al.

Julia G. Bodnarik, Dave K. Hamara, Arnold Burger, Vladimir Buliga, Joanna C. Egner, Michael Groza, Walter M. Harris, Liviu Matei, Keivan G. Stassun, Ashley C. Stowe, "Neutron detector development for microsatellites," Proc. SPIE 10392, Hard X-Ray, Gamma-Ray, and Neutron Detector Physics XIX, 103920M (29 August 2017); doi: 10.1117/12.2275682

EDent: SPIE Optical Engineering + Applications, 2017, San Diego, California, United States 


\title{
Neutron Detector Development for Microsatellites
}

Julia G. Bodnarik*a , Dave K. Hamara ${ }^{\mathrm{a}}$, Arnold Burger ${ }^{\mathrm{b}}$, Vladimir Buliga ${ }^{\mathrm{b}}$, Joanna C. Egner ${ }^{\mathrm{b}}$, Michael Groza ${ }^{b}$, Walter M. Harris ${ }^{\mathrm{a}}$, Liviu Matei ${ }^{\mathrm{b}}$, Keivan G. Stassun ${ }^{\mathrm{c}}$, Ashley C. Stowe ${ }^{\mathrm{d}}$; ${ }^{\mathrm{a}}$ Lunar and Planetary Laboratory, University of Arizona, 1629 E University Blvd, Tucson, AZ, USA 857210092; 'bept. of Physics, Fisk University, $100017^{\text {th }}$ Avenue N., Nashville, TN, USA 37208, ${ }^{\mathrm{C}}$ Vanderbilt University, Dept. of Physics and Astronomy, 6301 Stevenson Science Center, Nashville, TN, USA, 37203, ' ${ }^{\mathrm{Y}}-12$ National Security Complex, P.O. Box 2009, Oak Ridge, TN, USA 378318245

\begin{abstract}
We present a preliminary design for a novel neutron detection system that is compact, lightweight, and low power consuming, utilizing the CubeSat platform making it suitable for space-based applications. This is made possible using the scintillating crystal lithium indium diselenide $\left({ }^{6} \mathrm{LiInSe}\right)$, the first crystal to include ${ }^{6} \mathrm{Li}$ in the crystalline structure, and a silicon avalanche photodiode (Si-APD). The schematics of this instrument are presented as well as the response of the instrument to initial testing under alpha, gamma and neutron radiation. A principal aim of this work is to demonstrate the feasibility of such a neutron detection system within a CubeSat platform. The entire endto-end system presented here is $10 \mathrm{~cm} \mathrm{x} 10 \mathrm{~cm}$ x $15 \mathrm{~cm}$, weighs 670 grams and requires $5 \mathrm{~V}$ direct current at 3 Watts.
\end{abstract}

Keywords: neutron detection instrument, CubeSat, scintillator, Si-APD, ${ }^{6} \mathrm{LiInSe}_{2}$, radiation

\section{INTRODUCTION}

There is a growing interest in the characterization of near-Earth objects such as the moon, asteroids, and planets. Characterization includes the investigation of these objects' bulk composition, in an effort to better understand the chemistry of the sub-surface regolith. This can place constraints on solar system formation theories as well as provide valuable information for commercial endeavors such as asteroid mining. Previous measurements have primarily given information regarding the composition of the top-most surface layer, on the order of about 10 centimeters [1]. While the characterization of the surface of objects is valuable, often the surface and bulk compositions are very different because of exposure and weathering effects that predominantly affect the surface such as ionization from the sun, the evaporation of volatiles due to low surface gravity or lack of atmosphere, and erosion from cosmic wind [2].

A lithium indium diselenide ( ${ }^{6} \mathrm{LiInSe}_{2}$ ) based CubeSat instrument will have the capability to probe to depths of 50 centimeters below the surface of solar system bodies, at a fraction of the weight and power of the traditional instruments described in the literature $[1,3,4,5,6]$. The use of neutron detectors will be a major component in studies of the chemical composition of asteroids and planets. Previously, all neutron-detecting instruments have been large and require high voltage, making them impractical for a CubeSat platform. Examples of neutron detectors on previous missions include, the 2001 Mars Odyssey mission utilizing a combination of Helium-3 tube detectors and a boron-loaded plastic scintillator detector, with a mass of $30 \mathrm{~kg}$ and power requirement of 32W, and the 2007 Gamma Ray and Neutron Detector (GRaND) on the Dawn mission to explore Ceres and Vesta with both boron-loaded plastic and lithium-loaded glass scintillators, as well as a semiconducting cadmium zinc telluride (CdZnTe) crystal array, and a mass of 10kg and $15 \mathrm{~W}$ power consumption $[4,5,7]$. While ${ }^{3} \mathrm{He}$ gas tubes are highly efficient neutron detectors due to a high cross-section for neutron detection, they are heavy, require high voltage, and are bulky. Similarly, most scintillator-based detectors require pairing with photomultiplier tube (PMT) systems, which are sensitive to magnetic fields, add significant mass, and increase power consumption. Therefore, there is great interest in developing a cost-effective space instrument with a solid-state neutron detector that is more compact, requires less power, and does not have sensitivity to magnetic fields.

${ }^{6} \mathrm{Li}$ has a large cross-section of 940 barns for thermal neutron capture, following the reaction ${ }^{6} \mathrm{Li}+{ }^{1} \mathrm{n} \rightarrow{ }^{7} \mathrm{Li}^{*} \rightarrow$ ${ }^{4} \mathrm{He}(2.056 \mathrm{MeV})+{ }^{3} \mathrm{H}(2.729 \mathrm{MeV})$. The Q-value, or the energy released, depends on the energy liberated following the neutron capture and in this case is $4.785 \mathrm{MeV}$ [8]. Traditionally, ${ }^{6} \mathrm{Li}$ is used as an absorber on semiconductors to increase

*bodnarik@lpl.arizona.edu; phone 1520 621-2012; fax 1222 555-876; lpl.arizona.edu

Hard X-Ray, Gamma-Ray, and Neutron Detector Physics XIX, edited by Arnold Burger, Ralph B. James,

Michael Fiederle, Larry Franks, Stephen A. Payne, Proc. of SPIE Vol. 10392, 103920M

(C) 2017 SPIE · CCC code: $0277-786 X / 17 / \$ 18 \cdot$ doi: 10.1117/12.2275682 
efficiency, but ${ }^{6} \mathrm{LiInSe}_{2}$ is the first semiconductor to have ${ }^{6} \mathrm{Li}$ in the structure of the crystal. It can be operated as either a semiconductor or a scintillator, but at the current state of development and purification the signal-to-noise ratio is improved by $42 \%$ in the scintillator mode of operation $[9,10,11]$, making this the preferred mode of operation for the CubeSat prototype.

The development of a ${ }^{6} \mathrm{LiInSe}_{2}$ [9] thermal neutron sensor is especially attractive because of its close spectral matching (510 nm emission) with newly developed silicon based photodetectors such as silicon avalanche photodiodes (Si-APD), and silicon photomultipliers ( $\mathrm{SiPM}$ ) that can be used in place of traditional photomultiplier tubes. These are much less bulky and heavy, and have the potential to be packaged into a compact, lightweight, low power detector system. Comparing models from Hamamatsu Photonics, their SiPM weighs 1g and their PMT weights 100g, and the SiPM occupies only $10 \%$ of the volume.

The prototype of a neutron detection CubeSat system and the preliminary performance tests are discussed and demonstrate the ability to deploy a neutron detection instrument in a compact footprint. ${ }^{6} \mathrm{LiInSe}_{2}$ has been reported to have a response to both neutrons and gamma rays [2]. We investigate ${ }^{6} \mathrm{LiInSe}_{2}$ in the laboratory to determine this response and see if we can separate thermal neutrons, epithermal neutrons, and gamma rays from one another in the spectrum by using moderating materials and digital signal processing. The results from our study will help us determine if ${ }^{6} \mathrm{LiInSe}_{2}$ is a useful detector for space and planetary science missions.

The prototype combines the innovative technology of ${ }^{6} \mathrm{LiInSe}_{2}$ and a Si-APD. This pairing eliminates the need for a conventional photomultiplier tube making it possible to package this instrument under the restrictions of the CubeSat program. Section 2 describes the design of the instrument and briefly discusses each component as well as the reasoning behind selection. Section 3 presents preliminary results and the basics of the instrument functions. Section 4 presents the data analysis and modelling of the experiments. Section 5 presents the conclusion of the presented work.

\section{NEUTRON CUBESAT INSTRUMENT DESIGN}

\subsection{CubeSat Design Specifications}

NASA's CubeSat Launch Initiative is a platform that allows researchers to compete for flight opportunities to conduct low cost space science experiments. Instruments can be from one to six units (U), with some sub-increments of 0.5 units available, with specific weight, size, and power requirements that can be found on www.cubesat.org. The criteria for a $1.5 \mathrm{U}$ include the size of $10 \mathrm{~cm} \times 10 \mathrm{~cm} \times 15 \mathrm{~cm}$, a weight limit of $2.00 \mathrm{~kg}$ (4.4 lbs), and requirement to run off the power supplied by onboard solar panels or batteries [12]. The 0.5U IRIS Deep Space Transponder CubeSat, built by JPL, has a power consumption of $26 \mathrm{~W}$ during full power transmission, and the 6U JPL MarCO, has a power consumption of $35 \mathrm{~W}$ $[13,14]$.

\subsection{CubeSat Design}

In the development of our instrument we have utilized a 1.5U CubeSat chassis. The block diagram of the instrument is shown in Figure 1. In this figure, the active area of the encapsulated ${ }^{6} \mathrm{LiInSe}_{2}$ crystal detector is oriented to be at the top. A thermal neutron interacts with the ${ }^{6} \mathrm{Li}$ nucleus, and the resulting nuclear reaction generates an energetic alpha particle that in an ionization cascade generates excited states in the scintillator crystal that ultimately recombine to their ground state by emitting optical photons [8]. The scintillating photons are then detected by the Si-APD and converted into an electronic signal, or pulse, that is further processed to generate a histogram of all events that constitute the spectrum of incoming radiation. In general, neutron detection takes place in a mixed field of other ionizing particles such as gamma and galactic cosmic rays, and the characteristics of the pulse can be used to discriminate between types of particles interacting with the detector.

The instrument has three stages, each comprised of a printed circuit board (labeled Boards 1, 2, and 3 in all figures) and the associated components. The top board contains the $12 \mathrm{~mm}$ x $12 \mathrm{~mm}$ x $2 \mathrm{~mm} 6 \mathrm{LiInSe} 2$ encapsulated crystal and the 11 x $11 \mathrm{~mm}$ Si-APD (components 1 and 2 in all figures), which are packaged in proper optical alignment in a light-tight fixture. Board 2 contains two DC-DC regulated power supplies (components 5 and 6 ) that convert the $5 \mathrm{~V}$ input to the instrument into $12 \mathrm{~V}$ and $440 \mathrm{~V}$ respectively. The $440 \mathrm{~V}$ power supply powers the Si-APD, while the $12 \mathrm{~V}$ supply runs the remaining components, the two amplifiers and the Kromek multichannel analyzer (MCA). 
1. Encapsulated LiInSe $\mathrm{C}_{2}$ Crystal

2. Silicon Avalanche Photo Diode

3. Assembly Housing

4. Room for microcomputer

5. 12V Power Supply

$6.440 \mathrm{~V}$ Power Supply

7. Multichannel Analyzer

8. Preamplifier

9. Shaping Amplifier

10. $5 \mathrm{~V}$ DC input, 3 watts
Incoming radiation

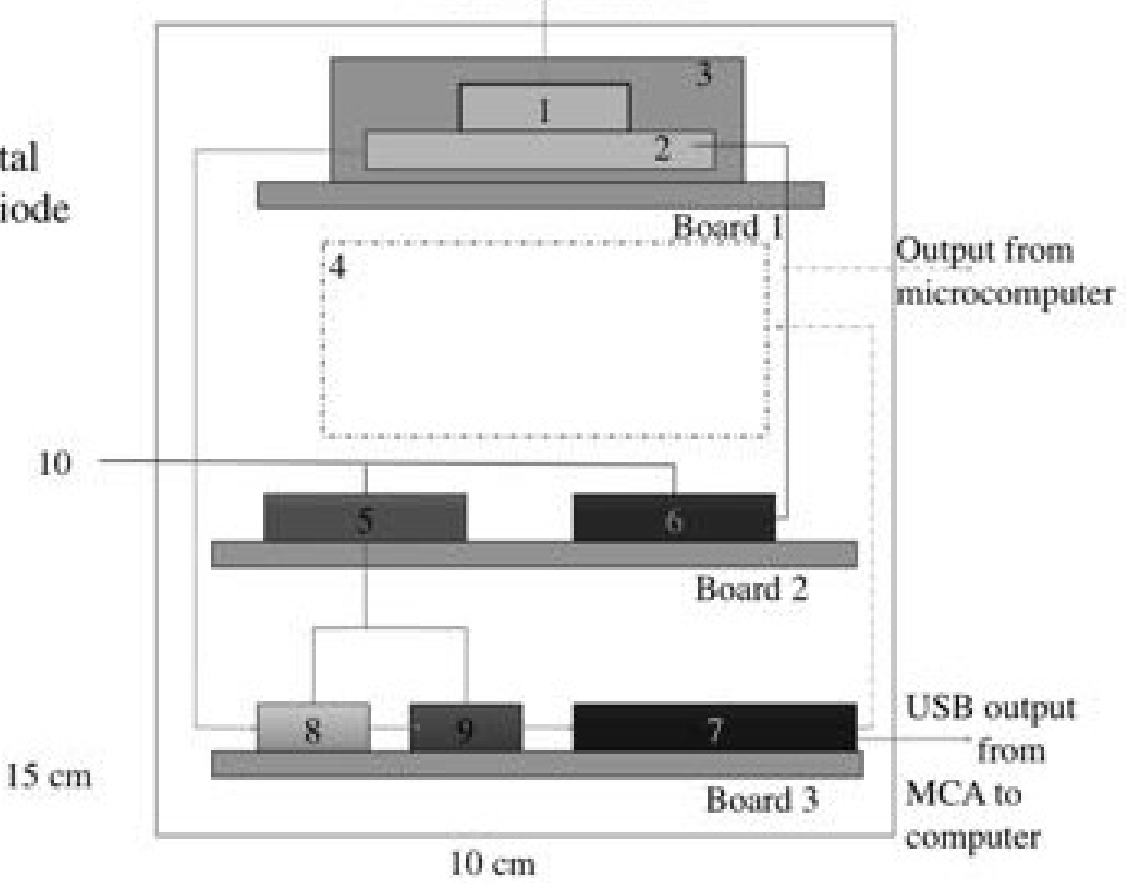

Figure 1: Neutron CubeSat prototype schematic.
The third board contains a preamplifier (component 8), a pulse shaping amplifier (component 9) and the MCA (component 7), which currently, for lab tests, outputs via USB cable to a computer. There is room as well as weight and power available for a microcontroller and wireless transmitter to be included in a later iteration of the instrument. A RaspberryPi was used in initial tests, and the current instrument, including the RaspberryPi microcontroller, weighs 670 grams in its entirety and requires $5 \mathrm{~V}$ and $3 \mathrm{~W}$.

The ${ }^{6} \mathrm{LiInSe}^{2}$ crystal used in the detector was grown using the vertical Bridgman method with details of growth being given by Tupytsin et al. and using a technology that was developed through a partnership between Fisk University and Y-12 National Security Complex [9]. ${ }^{6} \mathrm{Li}$ is a high-density material suitable for producing lightweight and compact neutron detectors due to its high capture cross section for thermal neutrons. ${ }^{6} \mathrm{Li}$ and other neutron sensitive materials are traditionally used as coatings on neutron detecting systems to increase the efficiency to a total of a few percent, in comparison, the density of ${ }^{6} \mathrm{Li}$ in ${ }^{6} \mathrm{LiInSe}_{2}$ great enough to yield up to $95 \%$ detection efficiency of neutrons in a $3.4 \mathrm{~mm}$ thick wafer [10].

The ${ }^{6} \mathrm{LiInSe}_{2}$ crystal utilized in the detector is $12 \mathrm{~mm} \times 12 \mathrm{~mm} \times 2 \mathrm{~mm}$. After characterization and testing, discussed more in section 3, it was encapsulated, covered on five sides with reflective material with a quartz window on the sixth side, shown in the topmost panel of Figure 2 labeled 1, this side was then placed onto the Si- APD.

The photo detector, in this case the Si-APD (component 2) turns detected events into electrical pulses. Compared to Si-APDs and silicon based photomultipliers (SiPMs), traditional vacuum tube photomultipliers (PMTs) are heavy, large, have high power requirements, and are sensitive to the magnetic environment of

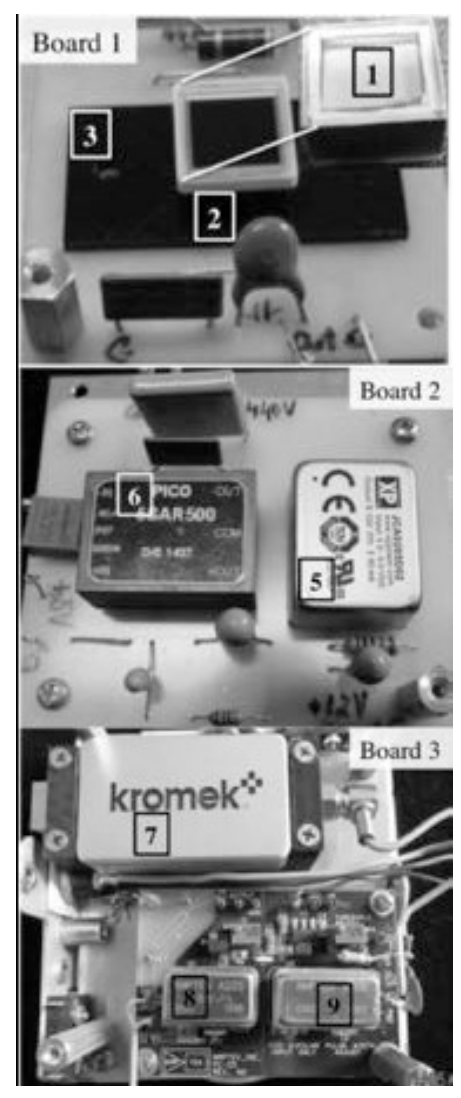

Figure 2: Photo of associated CubeSat electronics. Labeling of components is the same as the one used in Figure1. 
space. For these reasons, the use of a silicon based photo detector is better suited for space applications. A Hamamatsu S8664 1010 Si-APD (component 2) was utilized in this instrument. APDs utilize the photoelectric effect to turn the scintillated light from the crystal into an electronic signal with a sharp rise and a milliseconds-long tail [8].

The preamplifier and shaper, here an Amptek A225 (component 8), receives the signal from the Si-APD, amplifies it, then differentiates it and shapes it into a Gaussian shape with a width of several microseconds, preserving the energy as the height of the pulse. Next the voltage amplifier, an Amptek 206 (component 9), amplifies the pulse to a level that utilizes the full dynamic range of the following stages. This pulse then goes to the multichannel analyzer (MCA), a Kromek K102 (component 7). The MCA measures the Gaussian peaks from the voltage amplifier by pulse height (with the energy value still represented by height - shown in Figure 3) and histograms the pulses by their energy creating an energy spectrum of the interacting events.

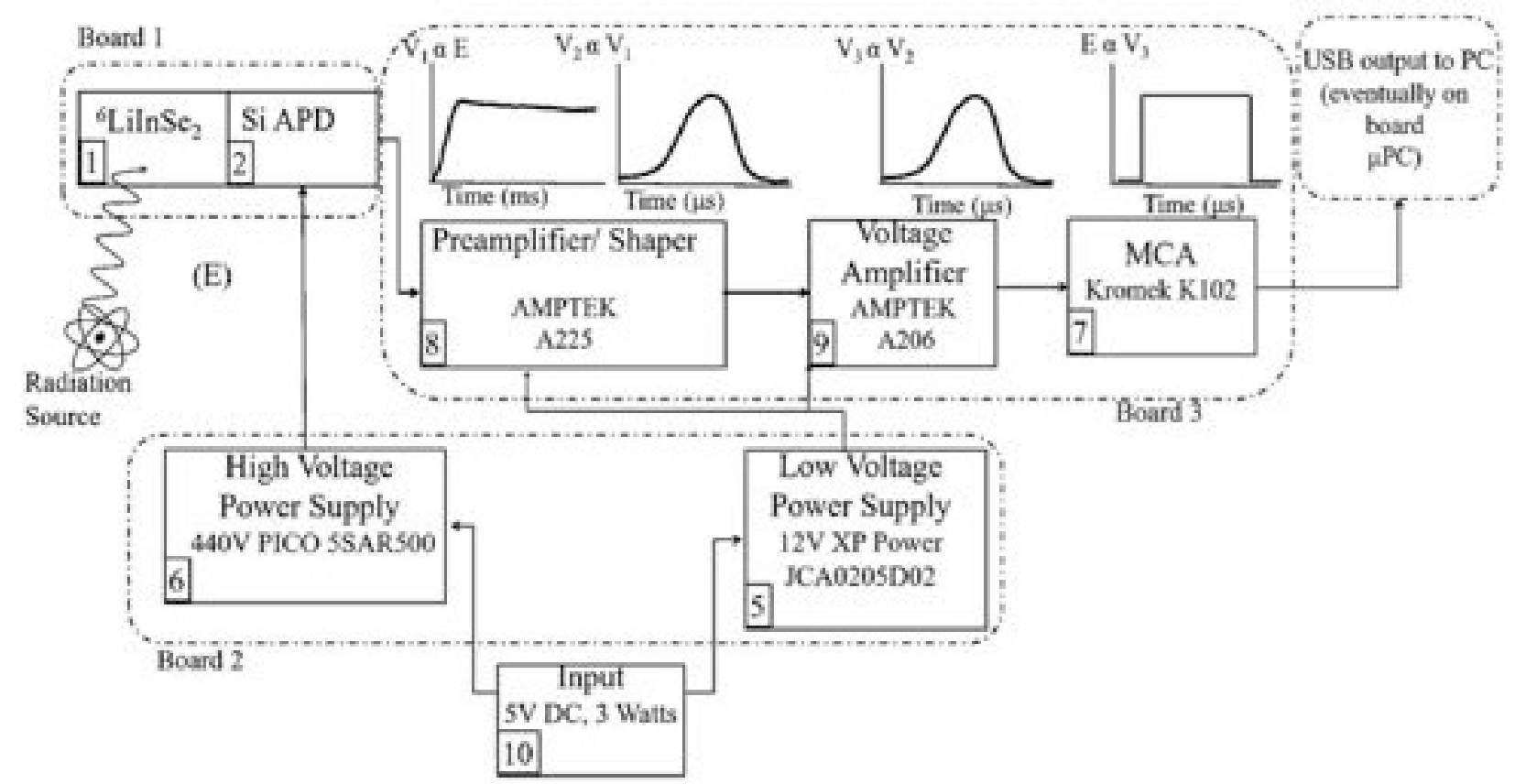

Figure 3: Block diagram of the detection system including ${ }^{6} \mathrm{LiInSe} 2$ crystal (1), Si-APD (2), associated electronics for signal processing $(7,8,9)$ and power supplies (5 and 6). The main information extracted from each signal is the height of the pulse, which is linearly proportional to the energy of the ionizing particle detected. Labeling of components is the same used in previous figures. The MCA measures the Gaussian peaks from the voltage amplifier by pulse height (with the energy value still represented by height - shown in Figure 3) and sorts pulses by their energy creating an energy spectrum of the interacting events.

\section{EXPERIMENTS AND DISCUSSION}

\section{$3.1{ }^{6}$ LiInSe2 $_{2}$ Crystal Scintillator}

The response to alpha particles is a good indication of the performance of the system before neutron testing. The ${ }^{6} \mathrm{LiInSe}_{2}$ crystal was fabricated into a scintillator and its response to alpha radiation from a ${ }^{241} \mathrm{Am}$ source with an activity of $0.9 \mu \mathrm{Ci}$ was tested at room temperature before encapsulation and integration into the instrument. The results are shown in Figure 4.

${ }^{241} \mathrm{Am}$ decays via the emission of an alpha particle with energy $5.486 \mathrm{MeV}$ to ${ }^{237} \mathrm{~Np}$ (Neptunium) [15]. The energy resolution of $36 \%$ was measured as the full width of the distribution at half the maximum of the peak (FWHM). This demonstrates a relatively high signal-to-noise (or signal-to-background) ratio of our system, indicating that we will be able to distinguish between two energies separated by $36 \%$ or more [8]. The low energy tail that can be seen in Figure 4 is due to general background, that includes the low energy, $60 \mathrm{KeV}$, gammas that are generated by this source. 


\subsection{Silicon Avalanche Photodiode (Si-ADP)}

Available scintillators emit in the blue or ultraviolet parts of the spectrum and therefore do not pair as well with siliconbased photo-detectors as ${ }^{6} \mathrm{LiInSe}_{2}$ does $[11,16,17]$. This is shown in Figure 5, where the quantum efficiency of the Hamamatsu S8664, given by the manufacturer, and the emission spectrum of a ${ }^{6} \mathrm{LiInSe}_{2}$ crystal, collected using X-ray excited optical luminescence, are shown. It can be seen that the ${ }^{6} \mathrm{LiInSe}_{2}$ crystal has its peak emission at about $510 \mathrm{~nm}$, and that the Si-APD has a high efficiency in this range. The blue or UV part of the spectrum, where most other scintillators emit, corresponds to the $400 \mathrm{~nm}$ range and lower. From 500 to $400 \mathrm{~nm}$, there is a drop of efficiency from 80 to $40 \%$ in the Si-APD. High efficiency in the photodetector is important because ${ }^{6} \mathrm{LiInSe}_{2}$ does not have a high light yield,

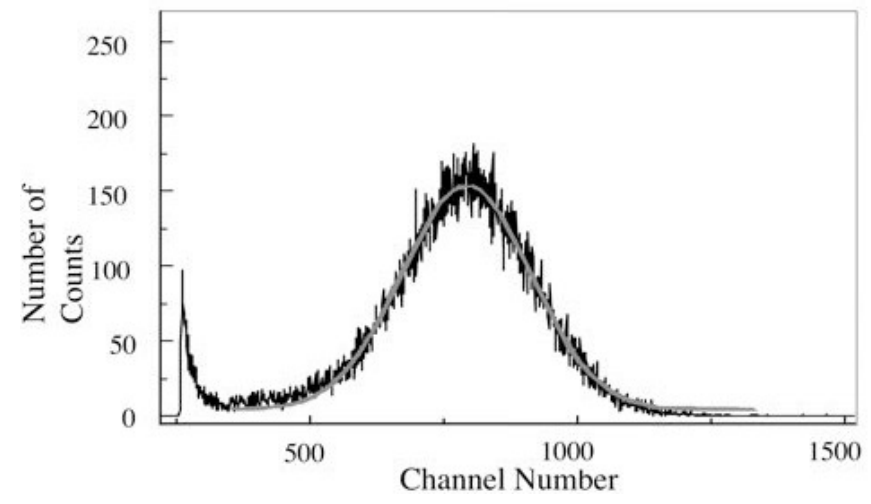

Figure 4: ${ }^{6} \mathrm{LiInSe}_{2}$ crystal response to alpha radiation from a ${ }^{241} \mathrm{Am}$ source. The spectrum was collected over 100 seconds.

4,400 photons $/ \mathrm{MeV}$ compared to inorganic and plastic scintillators which have light yields in the 10,000s (for example CsI(Tl) has a light yield of 65,000 photons/MeV) $[8,11]$.
LilnSe $e_{2} \mathrm{X}$-ray Excited Optical Luminescence

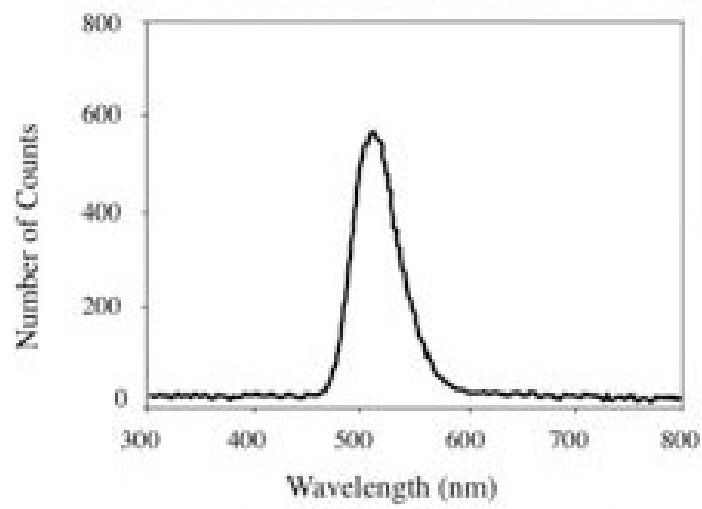

Quantum Efficiency of a Hamamatsu S8664

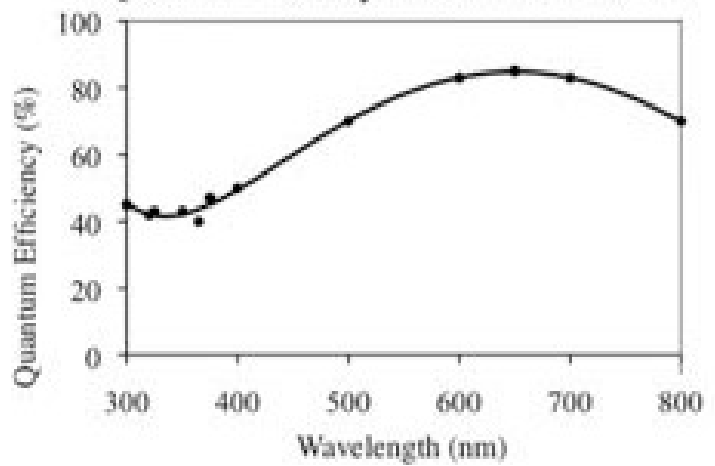

Figure 5: Comparison of Quantum Efficiency of a Hamamatsu S8664 1010 Si-APD and the output spectra of a ${ }^{6} \mathrm{LiInSe}_{2}$ crystal.

\subsection{Post-assembly System Test}

We have performed six radiation experiments with the ${ }^{6} \mathrm{LiInSe}_{2}$ CubeSat in varying neutron, gamma ray, and mixed radiation environments with differing moderating materials to determine if it is possible to separate neutrons from gamma rays in the resultant energy spectrum, since the detector is known to have a gamma ray and neutron response.

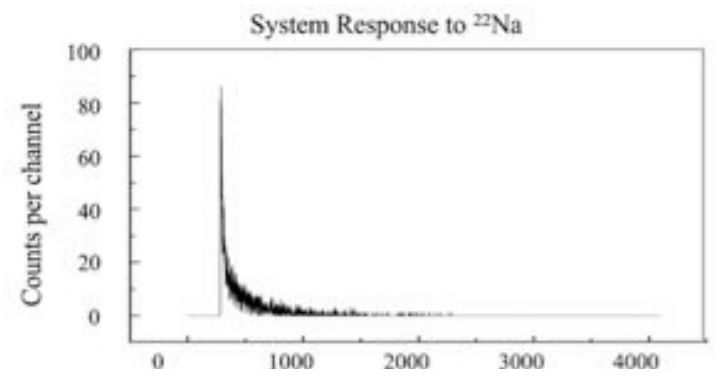

Figure 6: Response of packaged instrument to gamma radiation from a ${ }^{22} \mathrm{Na}$ source. Of the 5095 total counts collected, $70 \%$ are from direct interaction with the SiAPD.
Once the instrument was built, it was tested under gamma radiation. After encapsulation the system was tested by measuring the response to the $1.2 \mathrm{MeV}$ gammas emitted by ${ }^{22} \mathrm{Na}$, the spectrum collected is shown in Figure 6. The height of the ${ }^{22} \mathrm{Na}$ gamma signal is significantly lower than the response measured to the alpha particles from a ${ }^{241} \mathrm{Am}$ source.

We performed five additional experiments to investigate ${ }^{6} \mathrm{LiInSe}_{2}$ in the laboratory to determine its response to a mixed radiation environment and see if we can separate thermal neutrons, epithermal neutrons, and gamma rays from one another in the spectrum by using moderating materials and digital signal processing. The results from our study will help us determine if 
${ }^{6} \mathrm{LiInSe}_{2}$ is a useful detector for space and planetary science missions. In our second experiment, we placed the ${ }^{6} \mathrm{LiInSe}_{2}$ CubeSat into an aluminum frame that is mounted to the top of a 55-gallon shipping container containing ${ }^{252} \mathrm{Cf}$ encased in water-enhanced polyester (WEP), as shown in Figure 7.

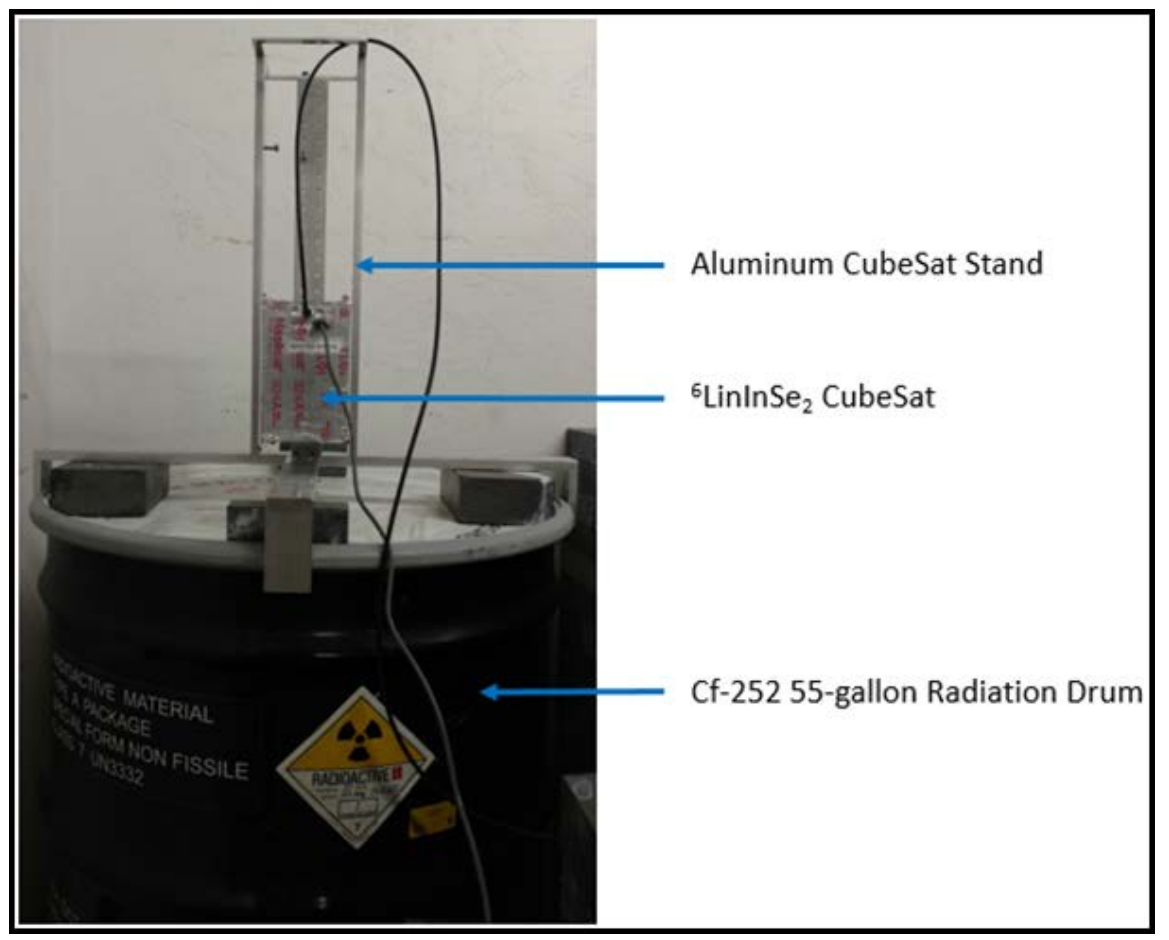

Figure 7. Image of experiment 2 . The ${ }^{6} \mathrm{LiInSe}_{2}$ CubeSat was placed in an aluminum holder on top of the bare ${ }^{252} \mathrm{Cf}$ barrel.

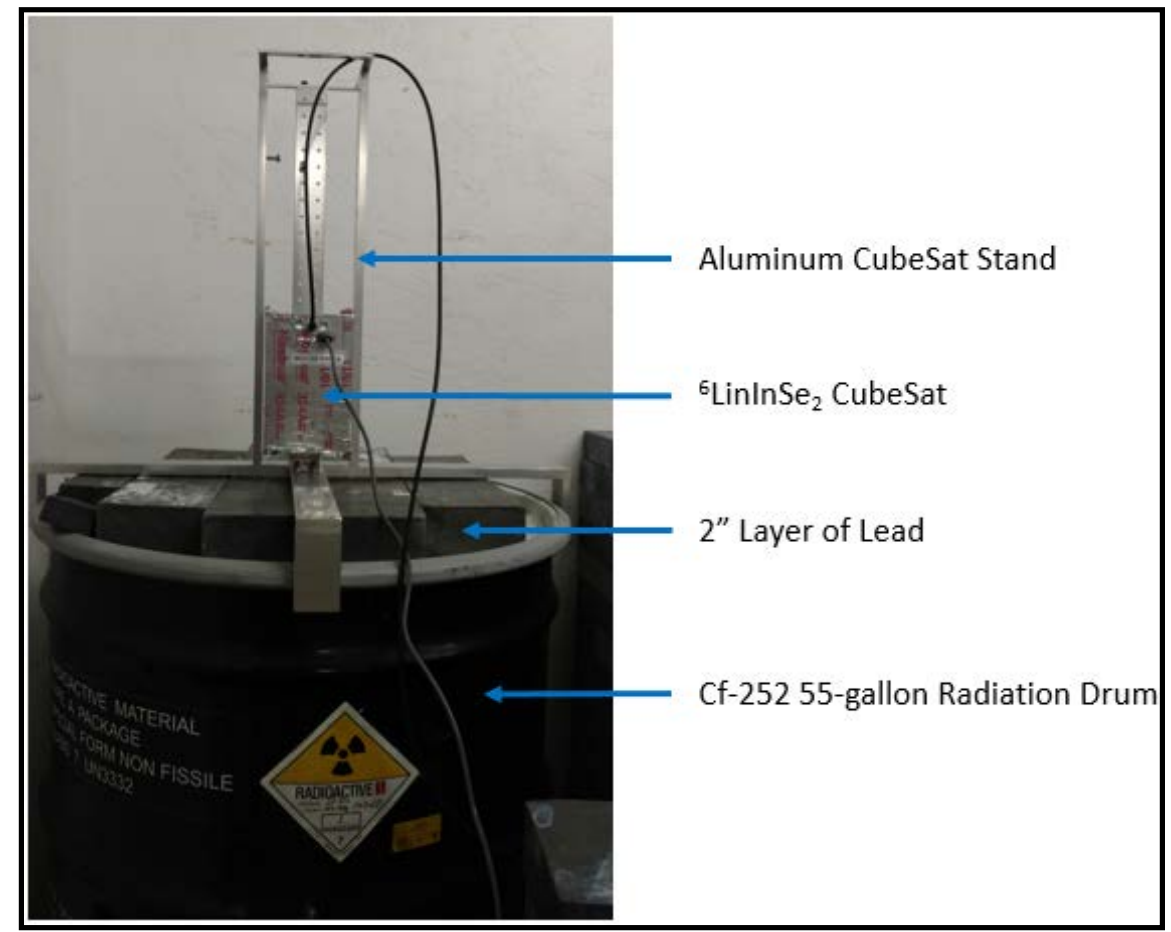

Figure 8. Image of experiment 3. The ${ }^{6} \mathrm{LiInSe}_{2}$ was placed in an aluminum holder on top of 2" thick lead shielding on top of the ${ }^{252} \mathrm{Cf}$ barrel. 
The ${ }^{6} \mathrm{LiInSe}_{2}$ detector was exposed for 2 hours to a mixed neutron and gamma ray radiation environment of moderated neutrons from ${ }^{252} \mathrm{Cf}$ and $2.2 \mathrm{MeV} \mathrm{H}$ gamma rays.

In the third experiment, we added a 2" thick layer of lead on top of the ${ }^{252} \mathrm{Cf}$ shipping container, as shown in Figure 8. The lead blocked out the gamma rays, leaving only the thermal and epithermal neutrons to reach the detector. The ${ }^{6} \mathrm{LiInSe}_{2}$ CubeSat was exposed for 2 hours to the neutron only radiation.

In the fourth experiment, we added a $2 \mathrm{~mm}$ thick layer of cadmium on top of the 2" layer of lead brick on top of the ${ }^{252} \mathrm{Cf}$ shipping container, as shown in Figure 9. The lead and cadmium shielded the detector from gamma rays and thermal neutrons, allowing only the epithermal neutrons to reach the detector. The ${ }^{6} \mathrm{LiInSe}_{2}$ CubeSat was exposed for 2 hours to the epithermal neutron radiation.

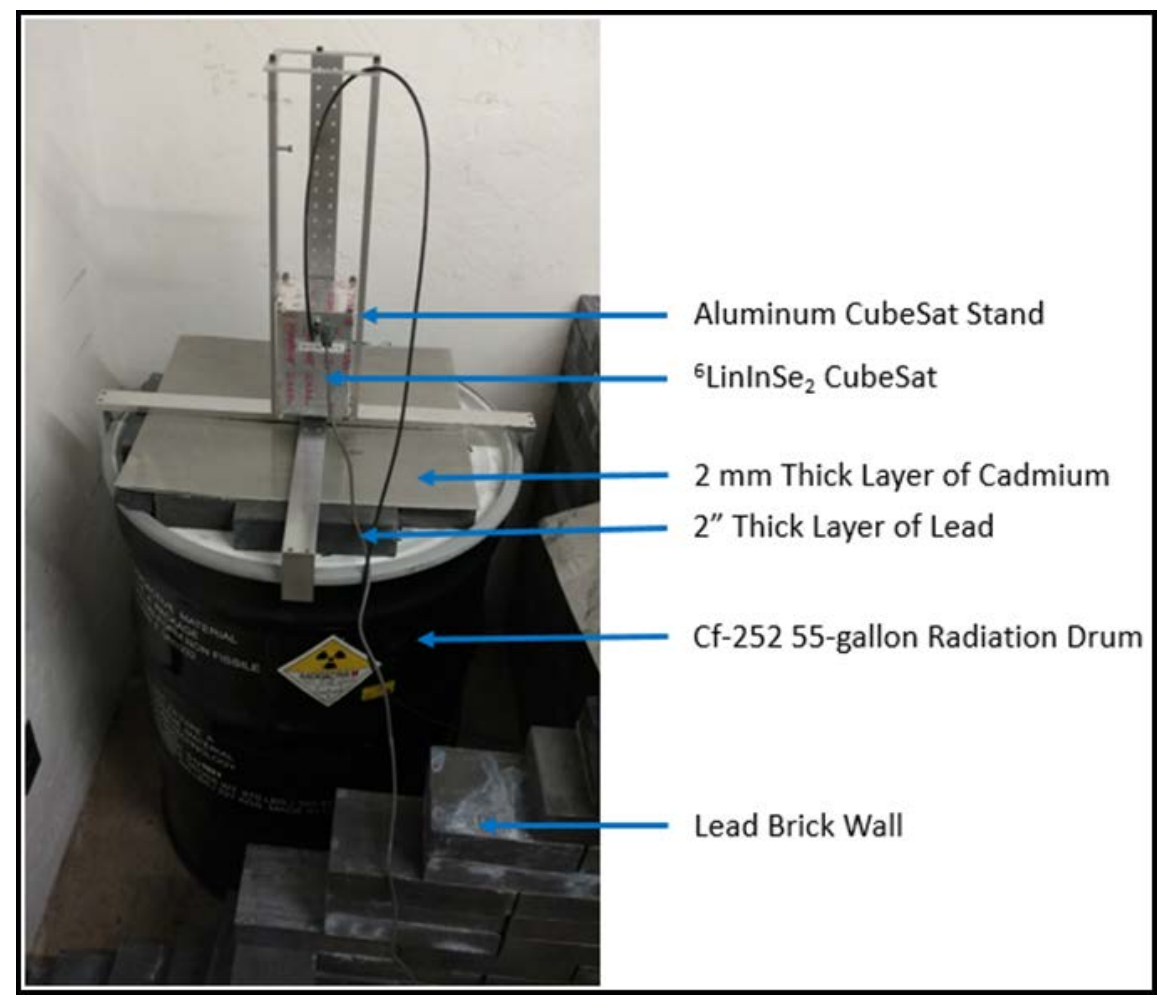

Figure 9. Image of experiment 4. The ${ }^{6} \mathrm{LiInSe}_{2}$ CubeSat was placed in an aluminum holder on top of a $2 \mathrm{~mm}$ thick layer of cadmium shielding on top of a 2" layer of lead shielding on top of the Cf-252 barrel. 
In the fifth experiment, we exposed the detector to only two gamma ray sources, ${ }^{60} \mathrm{Co}$ and ${ }^{152} \mathrm{Eu}$, as shown in Figure 10. The ${ }^{6} \mathrm{LiInSe}_{2}$ CubeSat was exposed for 24 hours to a gamma ray radiation from ${ }^{60} \mathrm{Co}$ and ${ }^{152} \mathrm{Eu}$. A 24 hour accumulation was necessary due to the weak sources and the poor efficiency of the detector for gamma radiation.

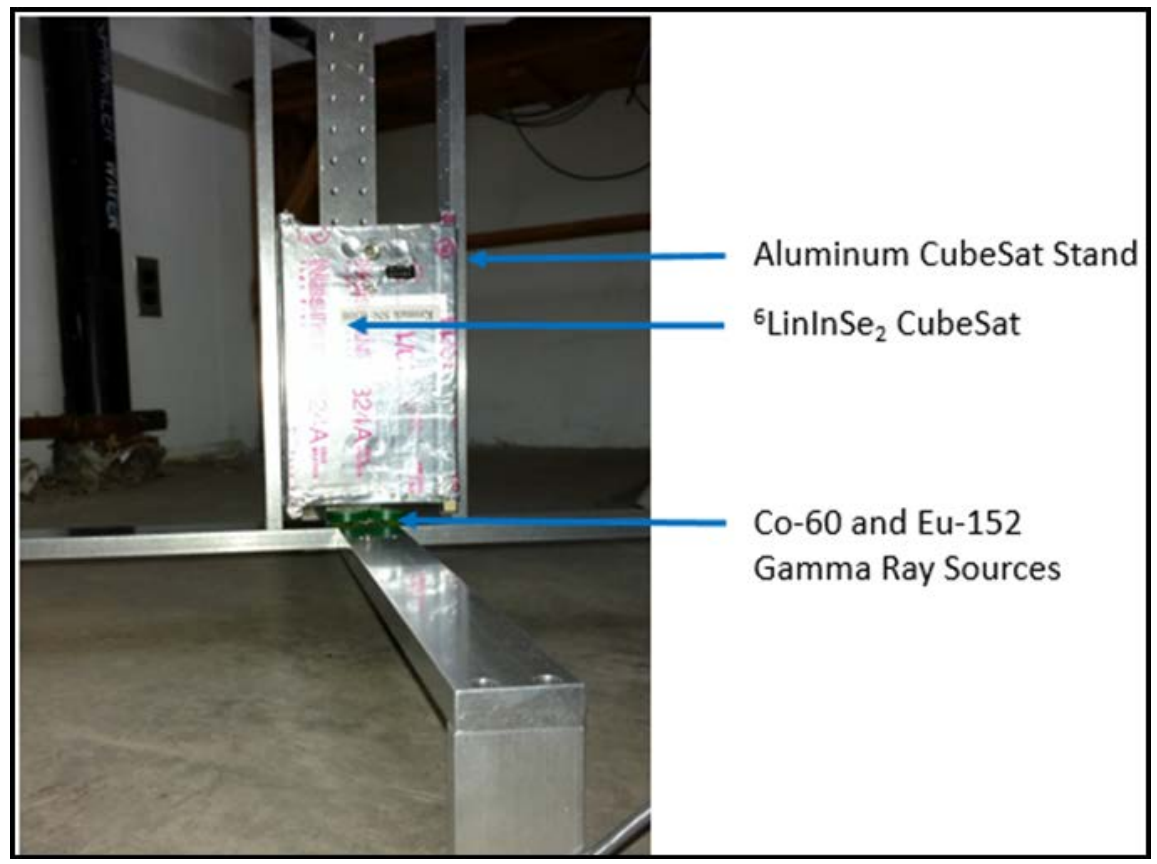

Figure 10. Image of experiment 5. The ${ }^{6} \mathrm{LiInSe}_{2}$ CubeSat was placed in an aluminum holder over two gamma ray sources, ${ }^{60} \mathrm{Co}$ and ${ }^{152} \mathrm{Eu}$.

In the sixth and final experiment, we took a background radiation spectrum, as shown in Figure 11. The ${ }^{6} \mathrm{LiInSe}_{2}$ CubeSat was exposed for 30 minutes to a background radiation environment.

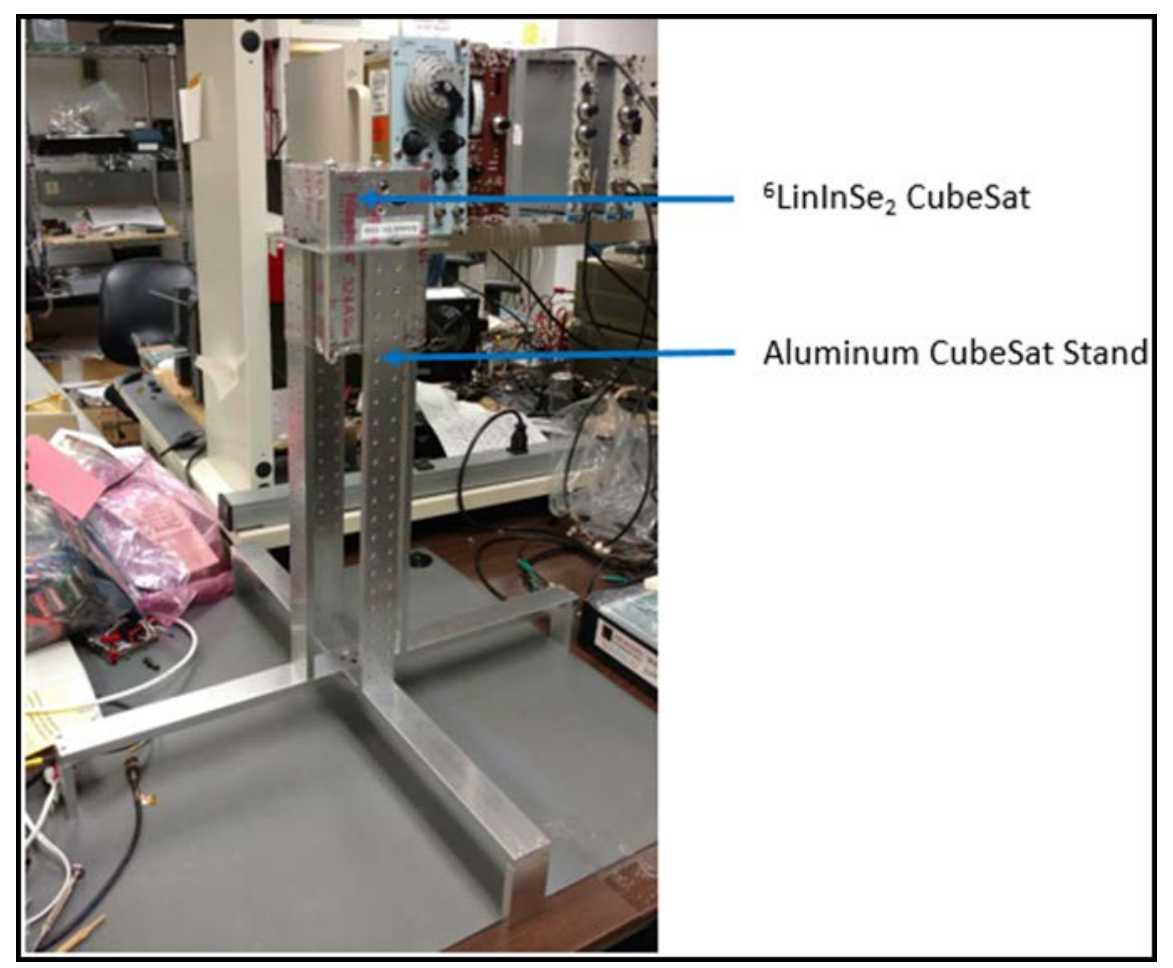

Figure 11. Image of experiment 6 . The ${ }^{6} \mathrm{LiInSe}_{2}$ CubeSat was placed in an aluminum holder with no sources present. 


\section{DATA ANALYSIS}

The data from the experiments was analyzed and fit using custom data analysis software written by Dave Hamara for the Mars Odyssey mission. Each fitted spectrum shows the fit, the area in counts under the associated fitted peaks and the residuals to the fits in sigma. Figures 12-17 show the results of the fitted gamma ray and neutron spectra. The full output for the fitted spectra is in Appendix I.

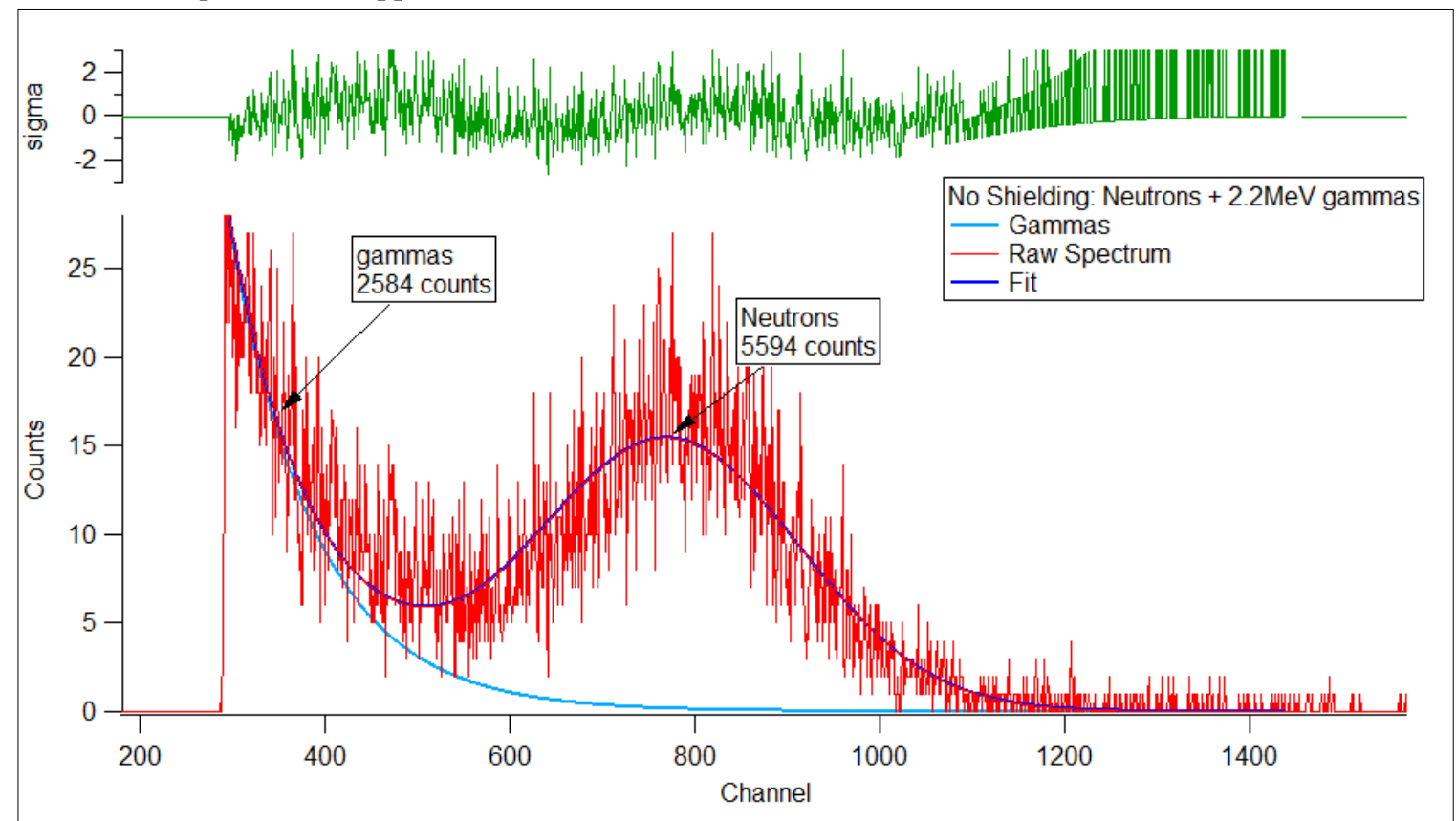

Figure 12. Neutron and gamma ray spectrum detected by the ${ }^{6} \mathrm{LiInSe}_{2}$ CubeSat for experiment 2 . The neutron and gamma ray spectrum data was reduced using custom software developed in Igor Pro, showing the residuals, in sigmas, and the fits to the gamma and neutron peaks.

Figure 12 shows the gamma ray and neutron spectrum for experiment 2, where the ${ }^{6} \mathrm{LiInSe}_{2}$ CubeSat was place in an aluminum holder on top of the bare ${ }^{252} \mathrm{Cf}$ barrel. The neutrons from ${ }^{252} \mathrm{Cf}$ were moderated from the water-enhanced polyester inside the barrel.

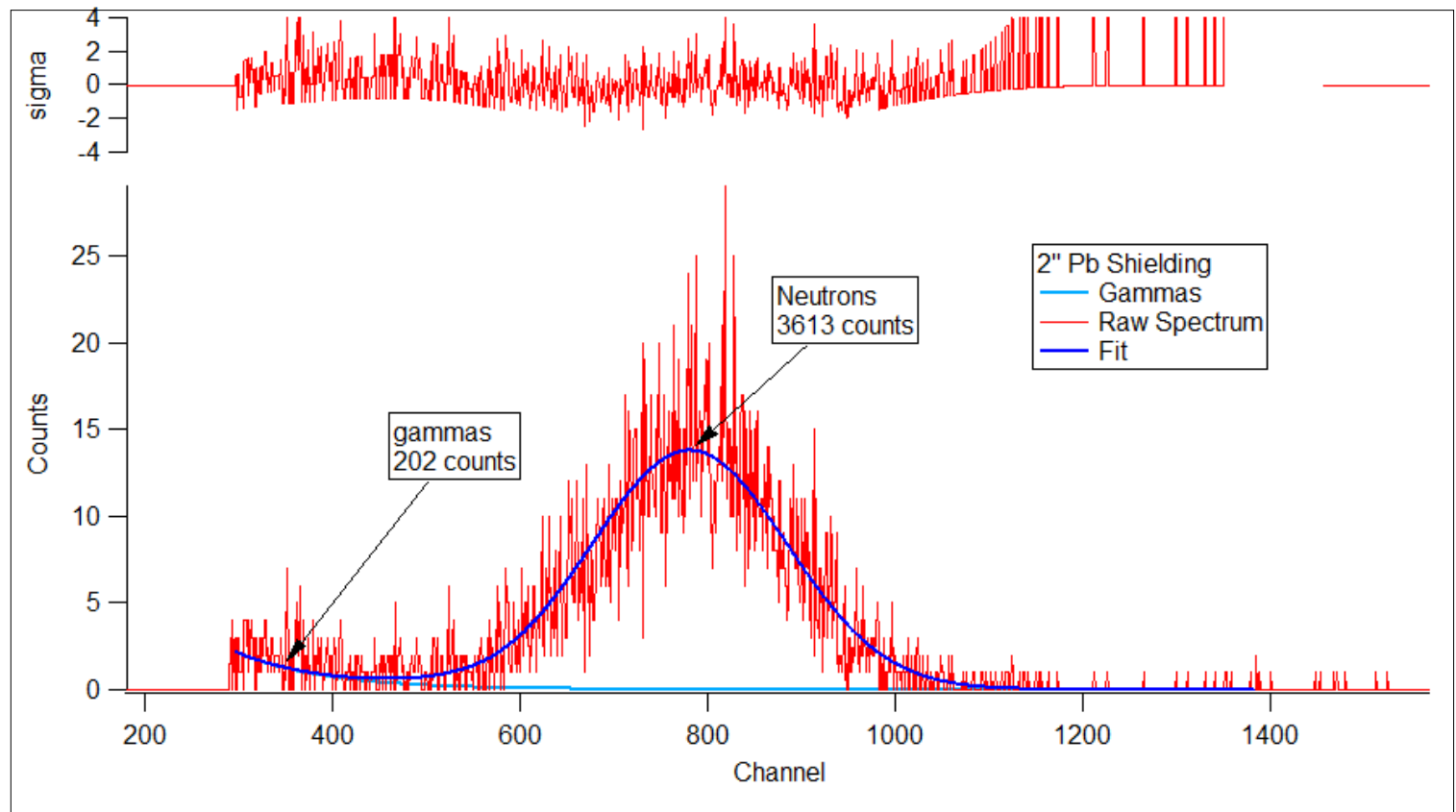

Figure 13. Neutron and gamma ray spectrum detected by the ${ }^{6} \mathrm{LiInSe}_{2}$ CubeSat for experiment 3 . The neutron and gamma ray spectrum data was reduced using custom software developed in Igor Pro, showing the residuals, in sigmas, and the fits to the gamma ray and neutron peaks. 
Figure 13 shows the gamma ray and neutron spectrum for experiment 4, where the ${ }^{6} \mathrm{LiInSe}_{2}$ CubeSat was placed in an aluminum holder on top of a 2" thick layer of lead shielding on top of the ${ }^{252} \mathrm{Cf}$ barrel. The ${ }^{252} \mathrm{Cf}$ neutrons were moderated from the water-ehnanced polyester (WEP) inside the barrel and the gamma rays were moderated by the 2" thick lead shielding. As you will notice in Figure 12, the gamma ray counts have been greatly reduced from 2584 counts in 2 hours, to 202 counts in 2 hours, as shown in Figure 13. The neutron counts have also been reduced from 5594 counts in 2 hours, as shown in Figure 12 to 3613 counts in 2 hours, as shown in Figure 13. This reduction in the neutron counts can be attributed to neutrons being blocked by the lead shielding.

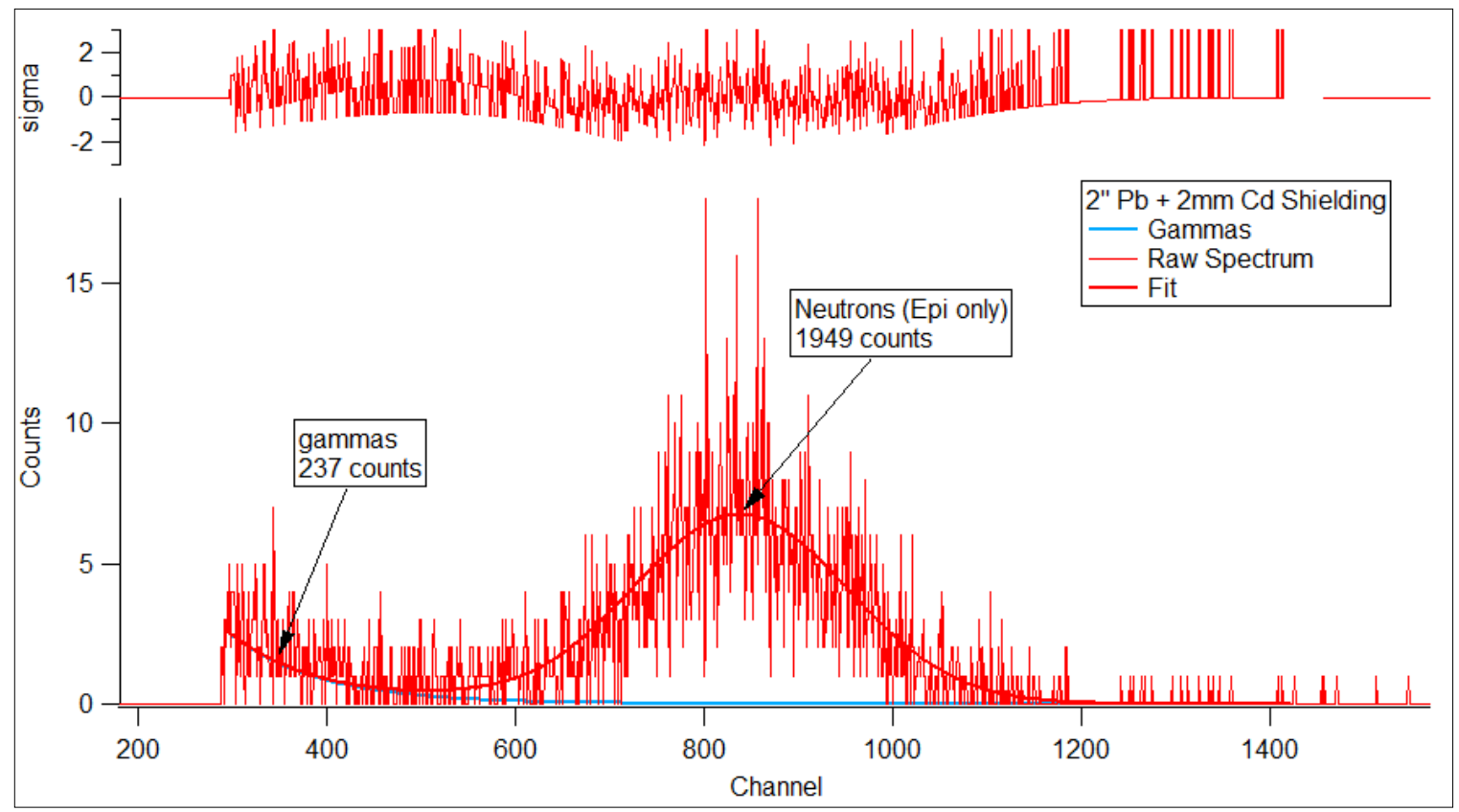

Figure 14. Neutron and gamma ray spectrum detected by the ${ }^{6} \mathrm{LiInSe}_{2}$ CubeSat for experiment 4 . The neutron and gamma ray spectrum data was reduced using custom software developed in Igor Pro, showing the residuals, in sigmas, and the fits to the gamma ray and neutron peaks.

Figure 14 shows the gamma ray and neutron spectrum for experiment 4 , where the ${ }^{6} \mathrm{LiInSe}_{2}$ CubeSat was placed in an aluminum holder on top of a $2 \mathrm{~mm}$ thick layer of cadmium shielding on top of a 2" thick layer of lead shielding on top of the ${ }^{252} \mathrm{Cf}$ barrel. The ${ }^{252} \mathrm{Cf}$ neutrons were moderated from the WEP inside the barrel and the $2 \mathrm{~mm}$ thick layer of cadmium shielding, and the gamma rays were blocked by the 2" thick lead shielding. As you will notice in Figure 14, the neutrons count have been reduced from 3613 counts, in Figure 13, to 1949 counts in Figure 14. The gamma ray counts have not changed significantly within counting statistics from 202 counts, in Figure 13, to 237 in Figure 14.

Figure 15 shows the neutron and gamma ray spectrum for experiment 5 , where the ${ }^{6} \mathrm{LiInSe}_{2}$ CubeSat was placed in an aluminum holder over 2 gamma ray sources, ${ }^{60} \mathrm{Co}$ and ${ }^{152} \mathrm{Eu}$, approximately 3 feet away from the neutron source in the barrel on the other side of the lead brick wall. The spectrum was collected for a 24 hour period. The gamma counts collected over the 24 hour period are 162104 counts. You will also notice in the inset graph in Figure 15 that we fit a thermal plus epithermal neutron peak of 7520 counts and a mystery peak of 5559 counts. 


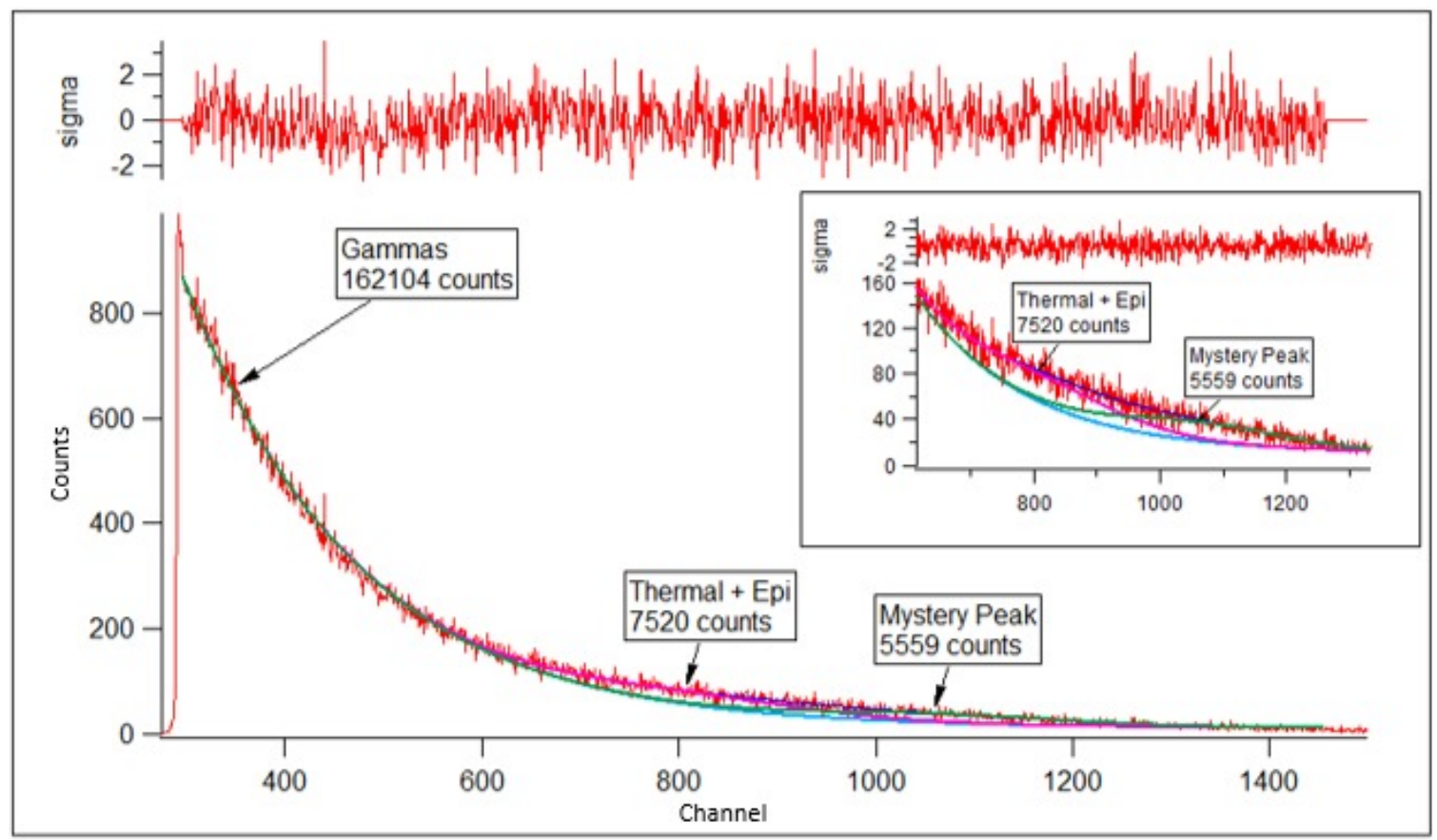

Figure 15. Neutron and gamma ray spectrum detected by the ${ }^{6} \mathrm{LiInSe}_{2} \mathrm{CubeSat}$ for experiment 5 . The neutron and gamma ray spectrum data was reduced using custom software developed in Igor Pro, showing the residuals, in sigmas, and the fits to the gamma ray and neutron peaks.

Figure 16 shows the background radiation spectrum for experiment 6, where the ${ }^{6} \mathrm{LiInSe}_{2}$ CubeSat was placed in an aluminum holder free from neutron and gamma ray sources. The background was collected over a 30 minute period. As you can see, the background count rate is very low at 1.2 counts per minute.

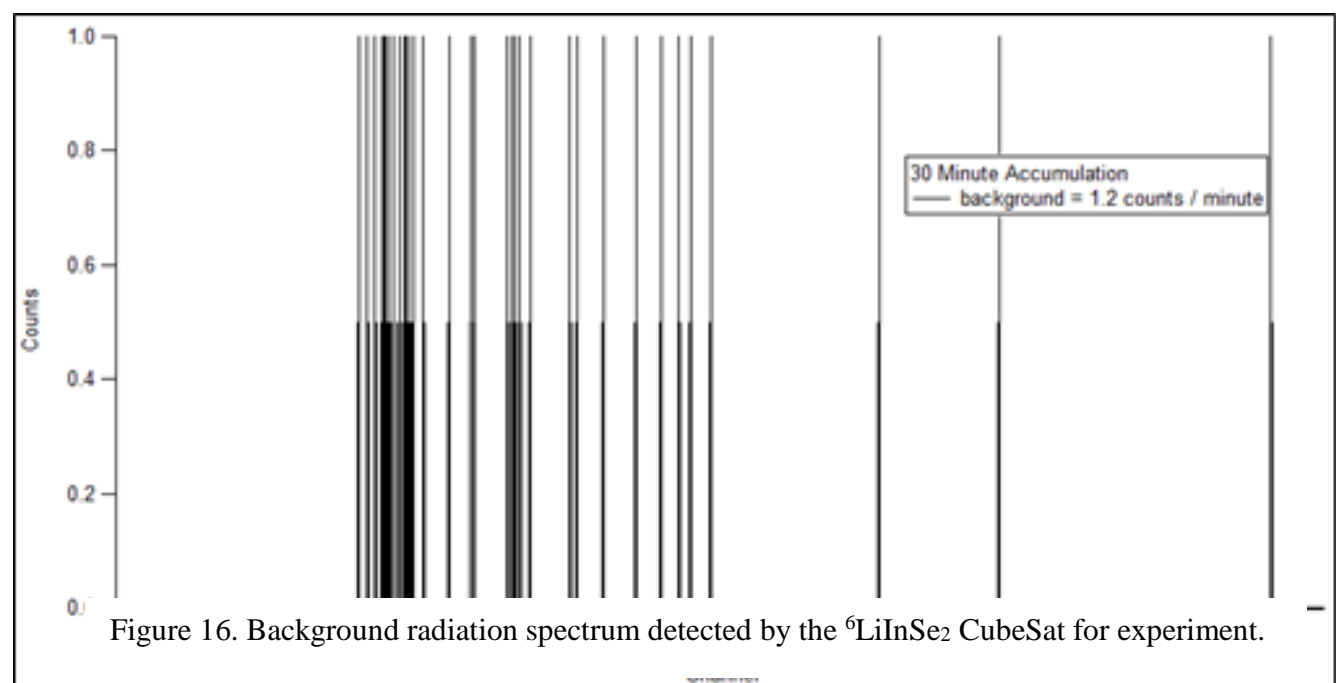

Figure 17 shows the results of the smoothed gamma ray and neutron spectrum from experiments 2-4. This figure qualitatively shows that we can separate gamma rays from WEP moderated thermal and epithermal neutrons using cadmium and lead shielding. 


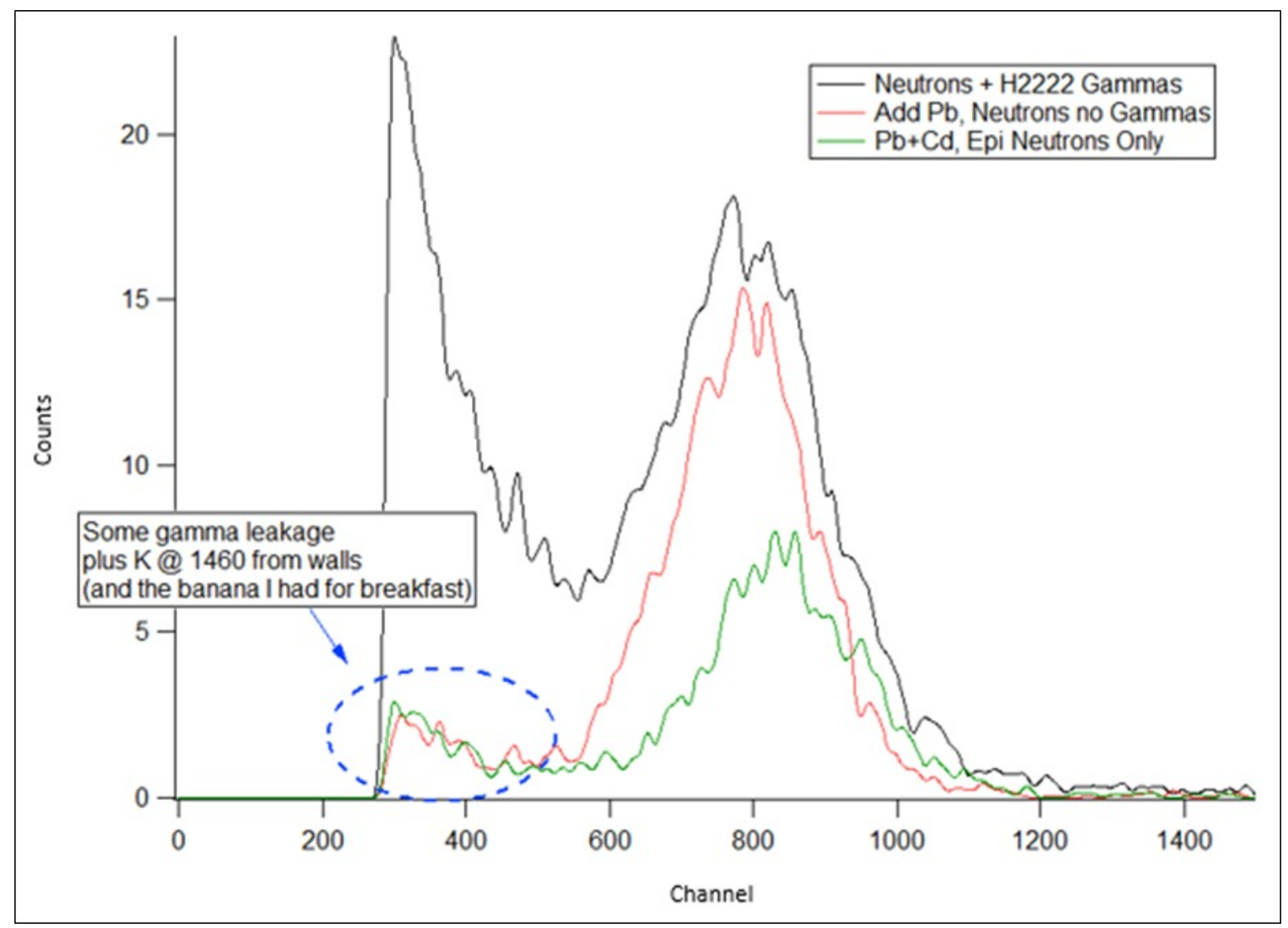

Figure 17. Smoothed neutron and gamma ray spectra from experiments 2-4.

Experiment 2-4 were modeled using the Monte Carlo Nuclear Particle eXtended code (MCNPX). The input files and output tallies are listed in Appendix II. The models were compared to the experimental data. Table 1 shows the model output and experimental data for experiments 2-4. Note that the MCNPX output data is for the flux of particles through the surface of the WEP barrel, the surface of the lead shielding and the surface of the cadmium shielding. The surface flux is normalized to the number of input particles and is in units of particles per square centimeter.

Table 1. MCNPX model output and experimental data results.

\begin{tabular}{|l|ll|lll|}
\hline \multicolumn{2}{|c|}{ MCNPX } & \multicolumn{3}{c|}{ Experimental Data } \\
\hline & & \multicolumn{3}{c|}{ Estimated Standard } \\
Exp. 2: WEP Barrel & Surface flux $/ \mathrm{cm}^{2}$ & Deviation of the Mean & Counts & Uncertainty & Time (sec) \\
\hline gammas & 32.35980 & 0.0046 & 2447 & $2.3 \%$ & 7200 \\
neutrons & 1.48783 & 0.0187 & 5594 & $1.7 \%$ & 7200 \\
\hline & & Estimated Standard & & & \\
Exp. 3: WEP \& Lead & Surface flux $/ \mathrm{cm}^{2}$ & Deviation of the Mean & Counts & Uncertainty & Time (sec) \\
\hline gammas & 1.78868 & 0.0175 & 202 & $8.9 \%$ & 7200 \\
neutrons & 0.95565 & 0.0284 & 3613 & $2 \%$ & 7200 \\
\hline & & Estimated Standard & & & \\
Exp. 4: WEP, Lead \& Cd & Surface flux $/ \mathrm{cm}^{2}$ & Deviation of the Mean & Counts & Uncertainty & Time (sec) \\
\hline gammas & 1.77696 & 0.0199 & 237 & $8 \%$ & 7200 \\
neutrons & 0.51760 & 0.0453 & 1949 & $2.5 \%$ & 7200 \\
\hline
\end{tabular}


Table 2 shows the ratios and ratio percentages for the MCNPX and experimental data results. With the exception of the MCNPX and the experimental data gamma ray ratios for the results of experiment 4 divided by experiment 3, the ratio percentages of the MCNPX and experimental data agree extremely well. We attribute the gamma results of the experiment 4 divided by experiment 3 to the poor counting statistics. The agreement shows that we are able to separate gamma rays from neutrons in our data analysis software, and thermal neutrons from epithermal neutrons using the appropriate shielding.

Table 2. MCNPX model output and experimental data results.

\begin{tabular}{|l|cl|ll|}
\hline Exp. 3/Exp. 2 & MCNPX Ratio & Percentage & Data Ratio & Percentage \\
\hline gammas & $5.527460 \mathrm{E}-02$ & $5.53 \%$ & 0.07817 & $7.82 \%$ \\
neutrons & $6.423080 \mathrm{E}-01$ & $64.23 \%$ & 0.64587 & $64.59 \%$ \\
\hline Exp. 4/Exp. 3 & MCNPX Ratio & Percentage & Data Ratio & Percentage \\
\hline gammas & $9.934505 \mathrm{E}-01$ & $99.35 \%$ & 1.173267327 & $117.33 \%$ \\
neutrons & $5.416271 \mathrm{E}-01$ & $54.16 \%$ & 0.539440908 & $53.94 \%$ \\
\hline
\end{tabular}

\section{CONCLUSION}

${ }^{6} \mathrm{LiInSe}_{2}$ is a highly attractive alternative neutron detector to ${ }^{3} \mathrm{He}$, due to the high neutron capture cross-section area of ${ }^{6} \mathrm{Li}$ (940 barns). Although the thermal neutron cross section of $6 \mathrm{Li}$ is lower that of ${ }^{3} \mathrm{He}$, the atomic density of ${ }^{6} \mathrm{Li}$ in a solid is higher, resulting in improved neutron absorption due to increased atomic density. This makes ${ }^{6} \mathrm{LiInSe}_{2}$ a promising candidate for thermal neutron detection in space science applications.

${ }^{6} \mathrm{LiInSe}_{2}$ has been reported to have a response to both neutrons and gamma rays [add references]. We investigate ${ }^{6} \mathrm{LiInSe}_{2}$ in the laboratory to determine this response and see if we can separate thermal neutrons, epithermal neutrons, and gamma rays from one another in the spectrum using moderating materials and digital signal processing if necessary. The results from our study will help us determine if ${ }^{6} \mathrm{LiInSe}_{2}$ is a useful detector for space and planetary science missions.

We have conducted five experiments, showing that we are able to successfully separate gamma rays from neutrons and thermal neutrons from epithermal neutron using appropriate shielding materials, such as WEP, lead and cadmium and custom post-processing data reduction software. ${ }^{6} \mathrm{LiInSe}_{2}$ will be a useful neutron detector for space-flight applications due to its small and compact size, low weight and low power requirements, making ideal for CubeSat missions and as a small instrument package for larger space missions.

\section{REFERENCES}

[1] J. O. Goldsten, “The NEAR X-Ray/Gamma-Ray Spectrometer,” Johns Hopkins APL Technical Digest, 19(2), 126-135 (1998).

[2] B. Wiggins, M.Groza, E. Tupitsyn, E. Lukosi, K. Stassun, A. Burger, A. Stowe. "Scintillation properties of semiconducting 6LiInSe2 crystals to ionizing radiation”. Nuclear Instruments and Methods in Physics Research Section A: Accelerators, Spectrometers, Detectors and Associated Equipment, 801, $73-77$ (2015).

[3] J.G. Bodnarik, D.M. Burger, A. Burger, L.G. Evans, A.M. Parsons, J.S. Schweitzer, R.D. Starr, and K.G. Stassun, "Time-resolved Neutron/Gamma-ray Data Acquisition for in situ Subsurface Planetary Geochemistry", Nuclear Instruments and Methods in Physics Research, A707, 135-142 (2013).

[4] W.V. Boynton, W.C. Feldman, I.G. Mitrofanov, L.G. Evans, R.C. Reedy, S.W. Squyres, R. Starr, J.I. Trombka, C. d'Uston, J.R. Arnold, P.A.J. Englert, A.E. Metzger, H. Wänke, J. Brückner, D.M. Drake, C. Shinohara, C. Fellows, D.K. Hamara, K. Harshman, K. Kerry, C. Turner, M. Ward, H. Barthe, K.R. Fuller, S.A. Storms, G.W. Thornton, J.L. Longmire, M.L. Litvak, and A.K. Ton'chev, "The Mars Odyssey Gamma-Ray Spectrometer Instrument Suite", Space Science Reviews, 110(1), 37-83(2004).

[5] W. C. Feldman, S. Maurice, A. B. Binder, B. L. Barraclough, R. C. Elphic, D. J. Lawrence, "Fluxes of Fast and Epithermal Neutrons from Lunar Prospector: Evidence for Water Ice at the Lunar Poles". Science, 281(5382), 1496-1500 (98). 
[6] A. Parsons, T. McClanahan, J.G. Bodnarik, L. Evans, S. Nowicki, J. Schweitzer, R. Starr, Subsurface In situ elemental composition measurements with PING, 2013 IEEE Aerospace Conference, (2013) 1-11.

[7] T. H. Prettyman, W. C. Feldman, H.Y. McSween Jr., R. D. Dingler, D. C. Enemark, D. E. Patrick, S. A. Storms, J. S. Hendricks, J. P. Morgenthaler, K. M. Pitman, and R. C. Reedy, “Dawn’s Gamma Ray and Neutron Detector”, Springer Science + Business Media B.V., 371-459 (2012).

[8] G.F. Knoll, Radiation Detection and Measurement. The United States of America John Wiley \& Sons, Inc., New Jersey (1979).

[9] E. Tupitsyn, P. Bhattacharya, E. Rowe, L. Matei, Y. Cui, V. Buliga, M. Groza, B. Wiggins, A. Burger, and A.Stowe, "Lithium Containing Chalcogenide Single Crystals for Neutron Detection". Journal of Crystal Growth. 393: 23-27(2014).

[10] Z. W. Bell, A. Burger, L. Matei, M. Groza, A. Stowe, J. Tower, A. Kargar, and H. Hong, "Neutron Detection in ${ }^{6} \mathrm{LiInSe}_{2}$ ", Proc. SPIE 9593, Hard X-Ray, Gamma-Ray, and Neutron Detector Physics XVII, 95930D, (2015).

[11]B. Wiggins, M.Groza, E. Tupitsyn, E. Lukosi, K. Stassun, A. Burger, A. Stowe. "Scintillation properties of semiconducting 6LiInSe2 crystals to ionizing radiation”. Nuclear Instruments and Methods in Physics Research Section A: Accelerators, Spectrometers, Detectors and Associated Equipment, 801, $73-77$ (2015).

[12] California Polytechnic State University CubeSat Program CubeSat Website: http://www.cubesat.org

[13] J. Nelson, T. Greicius, L. Espinoza, and K. Orr. JPL http://www.jpl.nasa.gov/cubesat/missions/iris.php

[14] A. Klesh,, J. Krajewski,, "MarCO: Cubesats to Mars in 2016,” Paper SSC15-III-3, 29th Annual AIAA/USU Conference on Small Satellites, Logan, UT, Aug. 10-13 2015.

[15] Radioactive Material Safety Data Sheet: Americium-241, Stuart Hunt \& Associates Ltd., St. Albert, Alberta, 2001.

[16] T. Saito, T. Nakamori, M. Yoshino, H. Mizoma, J, Kataoka, K. Kawakami, Y. Yatsu, M. Ohno, K. Goto, Y. Hanabata, H. Takahashi, Y. Fukazawa, M. Sasano, S. Torii, H. Uchiyama, K. Nakazawa, K. Makishima, S. Watanabe, M. Kokubun, T. Takahashi, K. Mori, H. Tajima, Astro-H HXI/SGD Team, Development of high performance Avalanche Photodiodes and dedicated analog systems for HXI/SGD detectors onboard the Astro$\mathrm{H}$ mission, Nuclear Instruments and Methods in Physics Research Section A: Accelerators, Spectrometers, Detectors and Associated Equipment. Volume 699, (2013) 230-234. 